\title{
La temperatura del pie como factor predictivo de aparición de úlceras en la Diabetes Mellitus
}

Raquel García de la Peña ${ }^{1}$; Salomón Benhamú Benhamú2; María Dolores Jiménez Cristino ${ }^{3}$; Jéssica Grande del Arco ${ }^{4}$; Gabriel Gijón Noguerón 5 .

Fecha de recepción: 14 de septiembre de 2018 / Fecha de aceptación: 15 de marzo de 2019.

Resumen. El Pie Diabético es una de las complicaciones más importantes de la Diabetes Mellitus debido a la posibilidad que existe de sufrir una úlcera plantar que aboque a una amputación.

La neuropatía periférica diabética acontece en un 60-70 \% de los sujetos con Diabetes Mellitus y se caracteriza por un trastorno de la sensibilidad táctil y dolorosa, la alteración del umbral de vibración y de la percepción térmica para los umbrales de temperatura al calor y al frío.

La temperatura de los pies de los sujetos con Diabetes Mellitus sin neuropatía periférica diabética es menor que con presencia de neuropatía periférica diabética. Se produce una correlación entre el aumento de la temperatura y la aparición de lesiones en los pies, debido a que la temperatura aumenta en las zonas de riesgo de ulceración por la propia inflamación y a la autolisis enzimática de los tejidos, previa a la aparición de una ulceración de la artropatía de Charcot. RESULTADOS: Actualmente se emplean dos tipos de técnicas de medición de la temperatura: termografía y termometría. Las pruebas de termografía se clasifican en placas superpuestas de cristal líquido termosensible e infrarrojos, y las pruebas de termometría se dividen en infrarrojos y los sensores térmicos.

La valoración térmica se debe incluir en los protocolos de exploración neurológica de los pies en la Diabetes Mellitus por ser una prueba eficaz, no invasiva y disminuye la tasa de aparición de úlceras.

CONCLUSIONES: Se considera que una diferencia de $2,2^{\circ} \mathrm{C}$, en el mismo punto de ambos pues de un sujeto, indica la aparición de lesiones propias del Pie Diabético, bien una ulceración si hay lesión, o una artropatía de Charcot.

La evidencia científica indica que el control de la temperatura de los pies disminuye la tasa de ulceraciones en la Diabetes Mellitus. Abogamos por la inclusión de la valoración térmica en el protocolo de exploración de los sujetos con Diabetes Mellitus, debido a su efectividad en la prevención de ulceraciones y por su escaso tiempo de cribado.

\section{[en] Foot temperature as a predictor of ulcers in Diabetes Mellitus}

Abstract. The Diabetic Foot is one of the most important complications of Diabetes Mellitus due to the possibility of suffering a plantar ulcer that leads to an amputation.

Diabetic peripheral neuropathy occurs in 60-70\% of subjects with Diabetes Mellitus and is characterized by a disturbance of tactile and painful sensitivity, the alteration of the threshold of vibration and thermal perception for temperature thresholds to heat and cold.

The temperature of the feet of subjects with Diabetes Mellitus without diabetic peripheral neuropathy is lower than with the presence of diabetic peripheral neuropathy. There is a correlation between the increase in temperature and the appearance of lesions on the feet, because the temperature increases in the areas of risk of ulceration due to the

\footnotetext{
1 Doctora por la Universidad de Sevilla. Profesora del Departamento de Podología de la Universidad de Sevilla. Facultad de Fisioterapia y Podología. Universidad de Sevilla. C/ Avicena /n. Sevilla, 41009. raquelgp@us.es

2 Doctor por la Universidad de Sevilla. Profesor del Departamento de Podología de la Universidad de Sevilla. benhamu@us.es

3 Diplomada en Podología por la Universidad de Sevilla. Profesora del Departamento de Podología de la Universidad de Sevilla. mjimenez45@us.es

4 Graduada en Podología. Universidad Complutense de Madrid. jessicagrandedelarco@gmail.com

5 Doctor por la Universidad de Málaga. Profesor de Podología de la Universidad de Málaga. gagijon8@gmail.com
} 
inflammation itself and to the enzymatic autolysis of the tissues, prior to the appearance of a ulceration of Charcot arthropathy.

RESULTS: Currently, two types of temperature measurement techniques are used: thermography and thermometry. Thermography tests are classified into thermo sensitive infrared liquid crystal plates, and the thermometry tests are divided into infrared and thermal sensors.

The thermal assessment should be included in the neurological examination protocols of the feet in Diabetes Mellitus because it is an effective, non-invasive test and decreases the rate of ulcer appearance.

CONCLUSIONS: It is considered that a difference of $2.2^{\circ} \mathrm{C}$, in the same point of both because of a subject, indicates the appearance of own injuries of the Diabetic Foot, either an ulceration if there is an injury, or a Charcot arthropathy. Scientific evidence indicates that controlling the temperature of the feet decreases the rate of ulcerations in Diabetes Mellitus. We advocate the inclusion of thermal assessment in the protocol of exploration of subjects with Diabetes Mellitus, due to its effectiveness in the prevention of ulcerations and its short time of screening.

Sumario: 1. Introducción.2. Material y método. 3. Relación entre la temperatura del pie y la neuropatía periférica diabética. 4. Resultados. Medición de la temperatura del pie. 4.1. Termografía. Imágenes térmicas infrarrojas. 4.2. Termografía. Cristales líquidos termográficos de contacto (LCT). 4.3. Termometría. Termómetro digital por infrarrojos. 4.4. Termometría. Báscula térmica. 4.5. Termometría. Termómetro con efecto Peltier. 5. ¿Por qué valorar la temperatura de los pies en la Diabetes Mellitus? 6. Conclusiones. 7. Bibliografía.

Cómo citar: García de la Peña, R. et ali (2019) La temperatura del pie como factor predictivo de aparición de úlceras en la Diabetes Mellitus, en Revista internacional de ciencias podólogicas 13(1), 115-129.

Los autores declaran no tener ningún tipo de interés económico o comercial.

\section{Introducción}

El síndrome del El Pie Diabético supone una de las complicaciones más importante y de mayor trascendencia clínica de la Diabetes Mellitus, ya que posee una gran repercusión en la población, debido a que a nivel mundial, es causa anualmente de la amputación de una pierna en más de un millón de personas ${ }^{1}$.

En España, en el período comprendido entre 2001 y 2008, se produjeron 90.064 amputaciones no traumáticas, de las cuáles el 64,5\% fueron debidas a la Diabetes Mellitus, estando relacionadas con la Diabetes Mellitus tipo 1 el 3,5\% de las mismas, mientras que el $61 \%$ restante se debió a la Diabetes Mellitus tipo 2.

Las úlceras que pueden producirse en los pacientes con Diabetes Mellitus se clasifican en neuropáticas, isquémicas o neuroisquémicas, teniendo en cuenta que se considera que en la Diabetes Mellitus es cuatro veces más probable presentar una arteriopatía periférica ${ }^{2}$.

La prevalencia de sujetos con Diabetes Mellitus que puede desarrollar una o más úlceras puede oscilar entre el $15-25 \%{ }^{3}$.

De los tres tipos de úlceras, salvo en las isquémicas, está presente la neuropatía periférica diabética, la cual se define como el conjunto de síntomas clínicos y subclínicos que señalan la presencia de disfunción neural en sujetos con Diabetes Mellitus ${ }^{4}$.

La Asociación Americana de Diabetes propuso, en el año 2008, que un sujeto presenta neuropatía periférica diabética si no percibe el monofilamento de Semmens - Weinstein 5.07 y unos de los siguientes signos: arreflexia, anestesia y apalestesia ${ }^{5}$ (Figura 1).

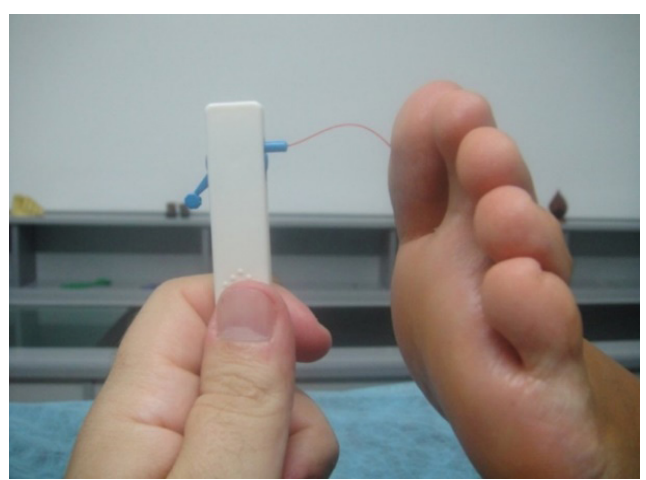

Figura 1: Aplicación del monofilamento de Semmes - Weinstein 5.07.

La neuropatía periférica diabética es muy frecuente en sujetos con Diabetes Mellitus, manifestándose aproximadamente entorno al $60-70 \%{ }^{4}$.

Desde el punto de vista de la fisiopatología de la neuropatía periférica diabética, los 
mecanismos que justifican su aparición se basan en la producción de metabólicos tóxicos y reactivos derivados de la hiperglucemia, de la presencia de microangiopatía e isquemia, de las anomalías en la señalización celular, de una desregulación a nivel del canal del sodio y de la presencia de edema axonal.

Estos mecanismos provocan una disminución de la velocidad de conducción nerviosa, un atrapamiento de los nervios, provocado por el edema que desarrolla la presencia de mononeuropatía en la Diabetes Mellitus $^{4} \mathrm{y}$, en último lugar, se produce una desmineralización segmentaria por obstrucción del vasa nervorum (arteriosclerosis y engrosamiento de su membrana basal) y glicosilación de la mielina (absorbida por los macrófagos) ${ }^{6}$.

La clínica característica de la neuropatía periférica diabética suele cursar con síntomas derivados de la alteración funcional de las fibras nerviosas, por exceso con presencia de parestesias y dolor urente, y por defecto se presenta con hipo o anestesia ${ }^{4}$.

La neuropatía periférica diabética se caracteriza también por un trastorno de la sensibilidad táctil y dolorosa y suele evolucionar de distal a proximal, siendo la presentación más frecuente de aparición la polineuropatía distal "en guante y/o calcetín"7.

Igualmente suele manifestarse mediante una alteración del umbral de vibración y percepción de la temperatura para los umbrales de temperatura al calor y al frío en manos y pies, al igual que la abolición de los reflejos rotuliano y aquíleo ${ }^{4}$.

\section{Material y método}

El presente trabajo tiene como objetivo sintetizar la evidencia disponible sobre la valoración de la temperatura como factor predictivo de aparición de úlceras en pacientes con Diabetes Mellitus.

Para ello hemos empleado revisiones sistemáticas sobre el tema sometido a estudio. Para ello se recurrieron a diferentes bases de datos y de recursos electrónicos (MEDLINE, PUBMED, SCOPUS y COCHRANE LIBRARY)

Los descriptores empleados durante la búsqueda de recursos bibliográficos fueron "temperature", "neuropathy", "thermometry", "thermography", "foot" y "Diabetes Mellitus".

Una vez obtenidos los artículos, resultado de la aplicación de dichos descriptores, y según el filtro correspondiente a los criterios de inclusión y de exclusión (tabla 1), obtuvimos doscientos dieciséis artículos. Una vez analizado íntegramente el contenido de cada uno de ellos, se seleccionaron finalmente cuarenta y dos, teniendo en cuenta que la información se ajustase al objetivo planteado en el presente estudio.

\begin{tabular}{|c|c|}
\hline Criterios de inclusión & Criterios de exclusión \\
\hline Revisión sistemática & Artículos no publicados \\
\hline Artículos en castellano y en inglés & \\
\hline El pie como elemento de estudio & $\begin{array}{c}\text { Neuropatías de etiología diferente a la } \\
\text { Diabetes Mellitus tipo } 1 \text { y } 2\end{array}$ \\
\hline $\begin{array}{c}\text { Neuropatía causada sólo por Diabetes Mellitus } \\
\text { tipo } 1 \text { y } 2\end{array}$ & \\
\hline
\end{tabular}

\section{Relación entre la temperatura del pie y la neuropatía periférica diabética}

Las primeras valoraciones de la temperatura en el pie en relación a la neuropatía periférica diabética, fueron realizadas por Goller et al ${ }^{8}$, que evaluaron por primera vez la temperatura de la piel como un marcador de inflamación y lesión en un pie con falta de sensibilidad.
Se considera que el aumento de temperatura de la piel de los pies se asocia a neuropatía periférica diabética, mientras que su descenso se relaciona con la presencia de alteraciones arteriales en el pie ${ }^{9}$.

Por tanto, existe una mayor temperatura en los pies de los sujetos con presencia de neuropatía periférica diabética, ya que en la neuropatía periférica diabética se 
produce un aumento del flujo sanguíneo a nivel de la microcirculación. Este hecho se justifica porque se disipa más calor por vasodilatación de los shunts arteriovenosos, los cuales dependen del control del nervio simpático ocasionando alteraciones de la termorregulación. Dicho aumento de la temperatura se produce tanto en la cara plantar como en la cara dorsal del pie, pero es en el dorso donde más aumenta la temperatura debido a la presencia de shunts arteriovenosos ${ }^{10}$ (Figura 2) (Tabla 4).

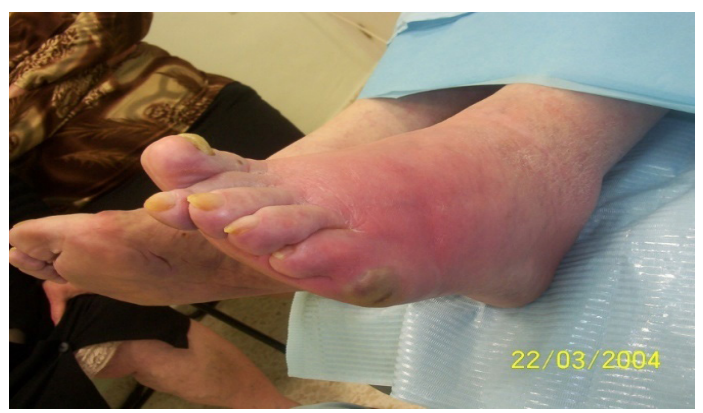

Figura 2: Aumento de la temperatura en ulcera infectada localizada en $5^{\circ}$ metatarsiano izquierdo.

Al contrastar los datos de algunas publicaciones sobre este hecho, se aprecia como la temperatura de los pies de los sujetos con Diabetes Mellitus control (sin presencia de neuropatía periférica diabética) es menor que la temperatura de los sujetos con Diabetes Mellitus que si presentan neuropatía periférica diabética (tabla 1).

\section{AUTORES}

Bagavathiappan et al, 2010 (9)

García-De-La-Peña et al, 2014 (11)

Chicharro et al, 2016 (1)

\section{$T^{a}$ podal DM control}

$27-30^{\circ} \mathrm{C}$

$26,8^{\circ} \mathrm{C}$

$30-31^{\circ} \mathrm{C}$

\section{$T^{a}$ podal DM NPD}

$32-35^{\circ} \mathrm{C}$

$28,2^{\circ} \mathrm{C}$

$32^{\circ} \mathrm{C}$

Tabla 1. Resumen de autores de la temperatura media del pie en sujetos control con Diabetes Mellitus y en sujetos con Diabetes Mellitus con neuropatía periférica diabética. Ta: Temperatura. DM: Diabetes Mellitus; NPD: Neuropatía periférica diabética.

Un segundo hecho a destacar es la valoración a realizar de la temperatura del mismo punto en ambos pies. Teniendo presente que la temperatura aumenta en las zonas de riesgo de ulceración, existe correlación entre el aumento de la temperatura y la aparición de una úlcera en el pie ${ }^{12}$ (Figura 3 ). 

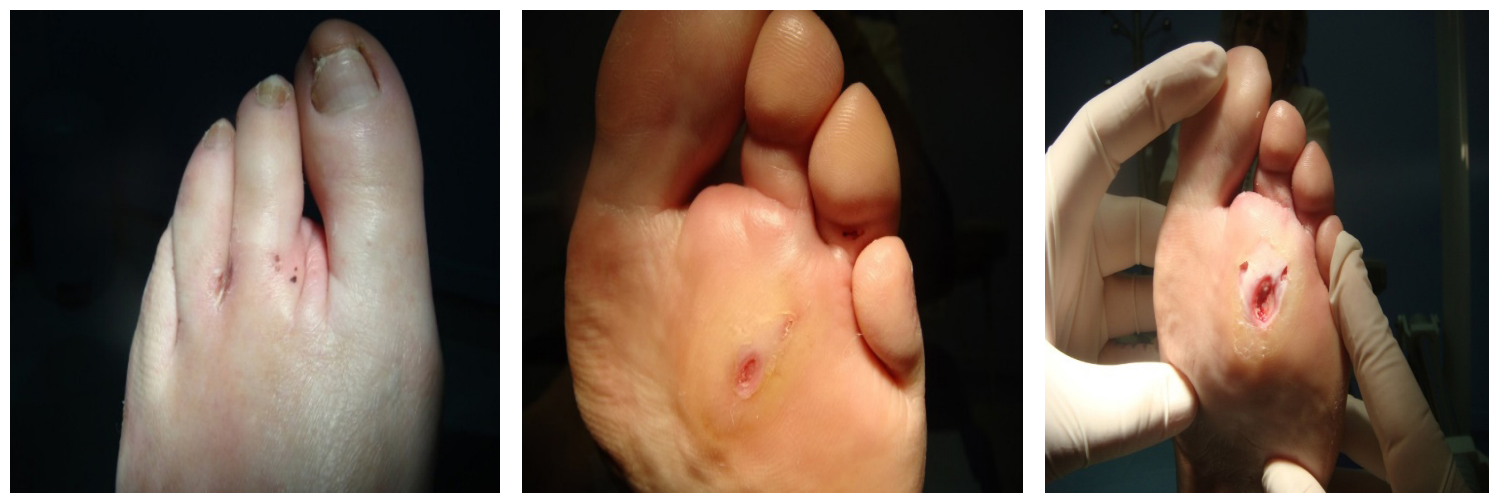

Figura 3. En la izquierda, paciente con neuropatía diabética y amputación del $2^{\circ}$ dedo izquierdo, con heridas por picaduras en zona interdigital y dorsal del $3^{\circ}$ dedo. En el centro y en la derecha, preulceración y ulceración, respectivamente, localizada en la cabeza del $3^{\circ}$ metatarsiano.

El aumento de la temperatura local se debe a la propia inflamación y a la autolisis enzimática de los tejidos, previa a la aparición de una ulceración ${ }^{13}$. También se produce el aumento de la temperatura en sujetos que presentan procesos inflamatorios propios de la artropatía de Charcot ${ }^{2}$.

Los valores de temperatura absoluta de los pies no se consideran los mejores indicadores por sí solos, ya que influyen factores tales como la edad, el género o la presencia de arteriopatías. Sin embargo, las diferencias de la temperatura entre un mismo punto de ambos pies sí son relevantes, al ser significativas a nivel clínico ${ }^{14}$.

En concreto, una diferencia de $2,2^{\circ} \mathrm{C}$ entre el mismo punto de ambos pies supone un importante/alto riesgo de aparición de lesión y/o inflamación inminente ${ }^{15}$. (Figura 4).
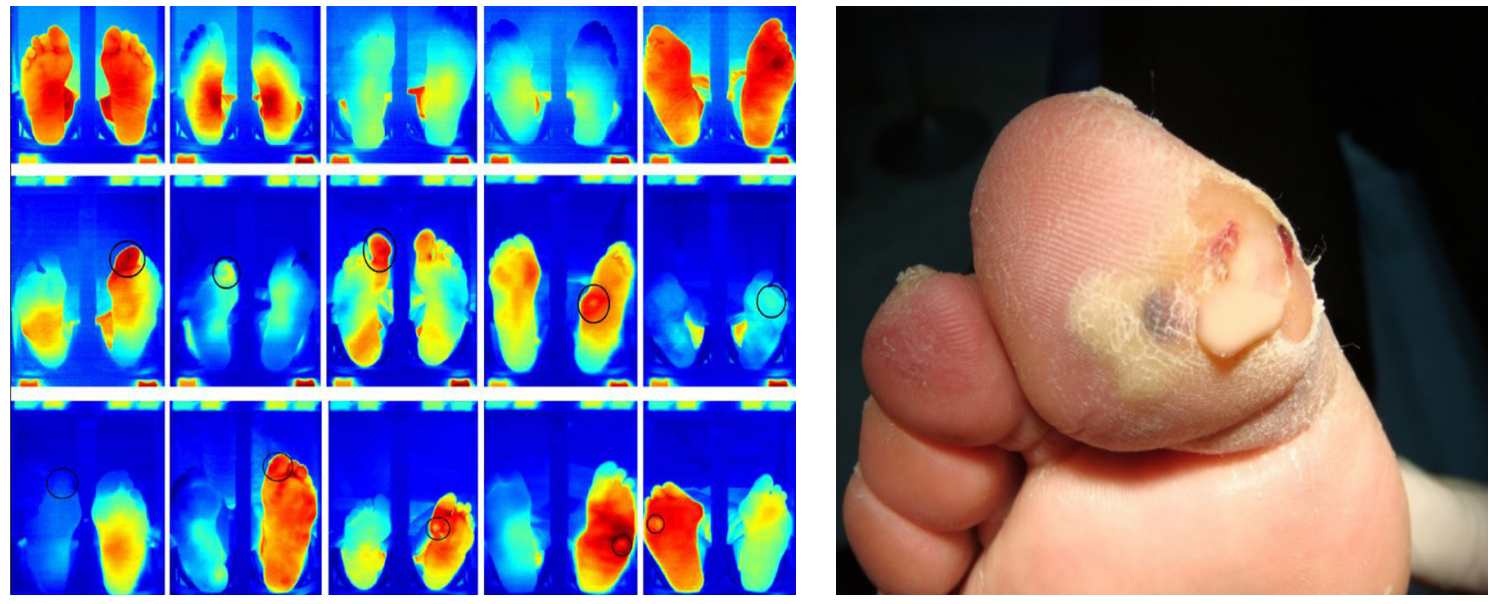

Figura 4: En la izquierda, imágenes térmicas de sujetos con patologías diversas en la que se observa un aumento de la temperatura en diferentes áreas, comparándolo ambos pies. Imagen tomada de Van Netten et al (2013). En la derecha, ulceración neuropática purulenta localizada en el pulpejo del $1^{\circ}$ dedo izquierdo, con aumento de la temperatura local, en una paciente diabética.

En el caso de detectar el aumento de temperatura mencionado anteriormente, se procede a disminuir la actividad física y a aplicar descargas selectivas hasta que la temperatura se normalice ${ }^{15}$ (Figura 5). 


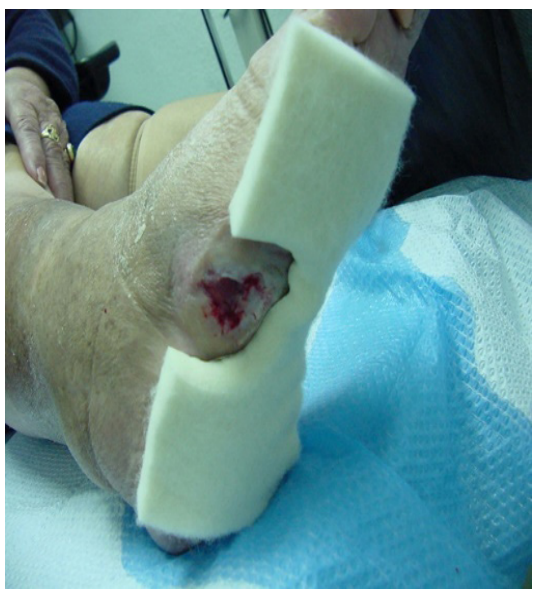

Figura 5. Aplicación de descarga selectiva en úlcera plantar en la apófisis estiloides del $5^{\circ}$ metatarsiano derecho.

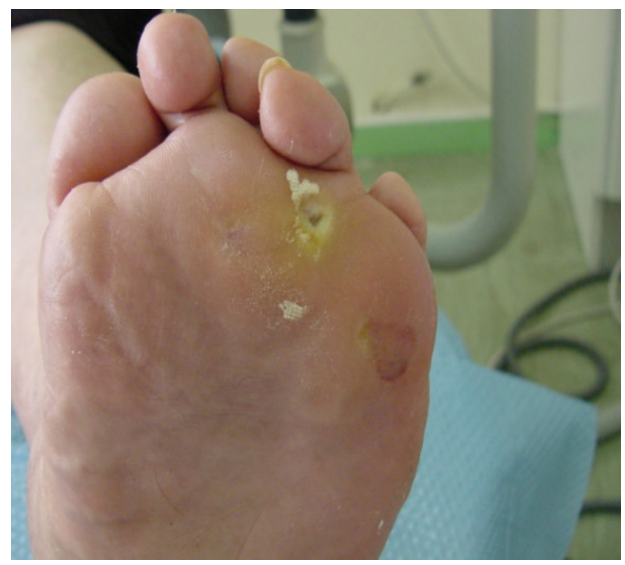

El aumento de la temperatura puede estar presente hasta una semana antes de que se produzca una úlcera ${ }^{16}$ (Figura 6).

Figura 6. En la izquierda, úlcera plantar en la cabeza del $4^{\mathrm{a}}$ metatarsiano izquierdo en tratamiento, con aumento de la temperatura plantar del $5^{\circ}$ metatarsiano izquierdo. En la derecha, cicatrización de la úlcera plantar en la cabeza del $4^{\mathrm{a}}$ metatarsiano izquierdo y aparición de ulceración en la cabeza del $5^{\circ}$ metatarsiano izquierdo.

Si se detecta una diferencia de temperatura mayor a $2,2{ }^{\circ} \mathrm{C}$ pero no existe herida/lesión dérmica en ese punto, hay que pensar en una artropatía aguda de Charcot $^{17}$ (Figura 7).

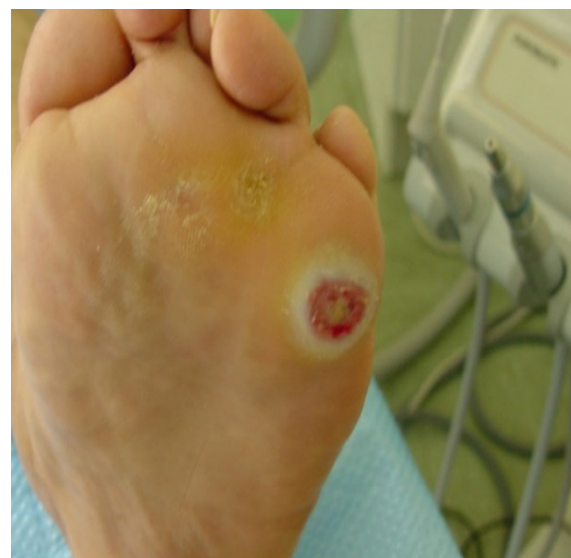


Además, las diferencias de temperatura se correlacionan con cambios radiológicos y con marcadores de remodelación ósea ${ }^{18}$.

El ascenso de temperatura suele ser demasiado sutil para ser detectado manualmente, por lo que se procede con su medición ${ }^{18-19}$.

\section{Resultados. Medición de la temperatura del pie}

Actualmente, se emplean tres sistemas de medición de la temperatura, los cuales se aplican mediante dos tipos de técnicas: la termografía y la termometría.

Las pruebas de termografía se clasifican en placas superpuestas de cristal líquido termosensible e infrarrojos.

Las pruebas de termometría se dividen en infrarrojos y los sensores térmicos ${ }^{20}$.

\subsection{Termografía. Imágenes térmicas infrarrojas.}

Proporcionan información de la distribución de la temperatura sobre toda la superficie plantar mediante una huella con un espectro de colores que representan las variaciones de temperatura ${ }^{20,21}$.

Para la interpretación de las termografías, Mori y colaboradores (2013) propusieron una clasificación de los patrones termográficos plantares. En ella, se sitúan los tipos 1 y 2 como valores normales; en el tipo 1 existe mayor temperatura en los arcos longitudinales internos, y en el tipo 2 existe una distribución completa de la temperatura ${ }^{20}$.

Los tipos 3 al 7 se consideran casos de valores con anomalías. Se ha de especificar que el tipo 3 corresponde a la "mariposa inversa", es decir, el tipo 1 pero con menor temperatura en los arcos longitudinales internos. Los casos que no se enmarquen dentro de estos patrones se consideran atípicos (Figura 8).

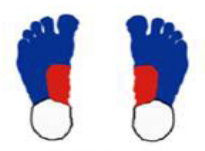

Type 1 Butterfly

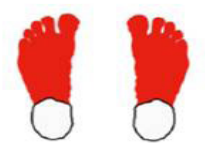

Type 2

Whole High

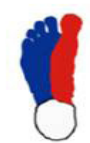

Type 4 Inner High

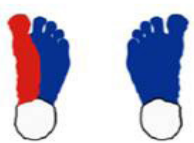

Type 5

Whole Low

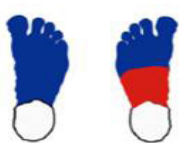

Type 6

Forefoot Low
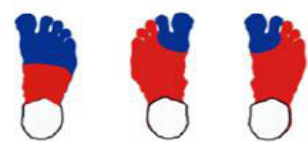

Type 7

Tiptoe Low

Figura 8. Patrones de imágenes térmicas infrarrojas según Mori et al (2013).

Aplicando las imágenes térmicas infrarrojas, Balbinot y colaboradores (2013) después de la inmersión de los pies en agua fría $\left(15^{\circ} \mathrm{C}\right)$ durante 10 minutos, observaron que el tiempo de respuesta de termorregulación es mayor en la neuropatía periférica diabética ${ }^{22}$.

Como principal ventaja, las imágenes térmicas infrarrojas aportan un mapa de la temperatura plantar, que permite una rápida valoración visual de ambos pies para analizar la temperatura de un mismo punto ${ }^{23}$.

Sin embargo, es necesario destacar que esta técnica precisa de instrumentos costosos (por ejemplo, cámaras específicas) y de equipos informáticos para el análisis de las imágenes, además de una formación especializada para su interpretación ${ }^{23}$.

\subsection{Termografía. Cristales líquidos termo- gráficos de contacto (LCT).}

Los cristales líquidos termográficos de contacto son placas indicadoras que están formadas por capas de cristales líquidos termográficos encapsulados. Se aplican en los pies, durante un minuto, para la obtención de imágenes térmicas.

Se genera una coloración proporcional a la temperatura de la zona valorada? .

Como principal inconveniente, hemos de resaltar la baja resolución de las imágenes que emiten. Sin embargo, es ventajoso el hecho de que la imagen se puede obtener tanto en estática como en dinámica ${ }^{24}$. 


\subsection{Termometría. Termómetro digital por infrarrojos.}

Respecto a la termometría, en primer lugar, destacamos el termómetro digital por infrarrojos. Es el método más empleado por la comunidad científica y por los pacientes, que lo emplean como autocontrol de la temperatura.
Se aplica perpendicular a la piel, a dos centímetros de distancia ya que no es un termómetro de contacto, en seis puntos de la planta del pie (primer dedo; cabezas de I, III, $\mathrm{V}$ metatarsianos; arco longitudinal externo y talón) $)^{2}$ (Figura 9).

$\mathrm{Su}$ diseño permite al usuario llegar a cualquier punto de la zona plantar².
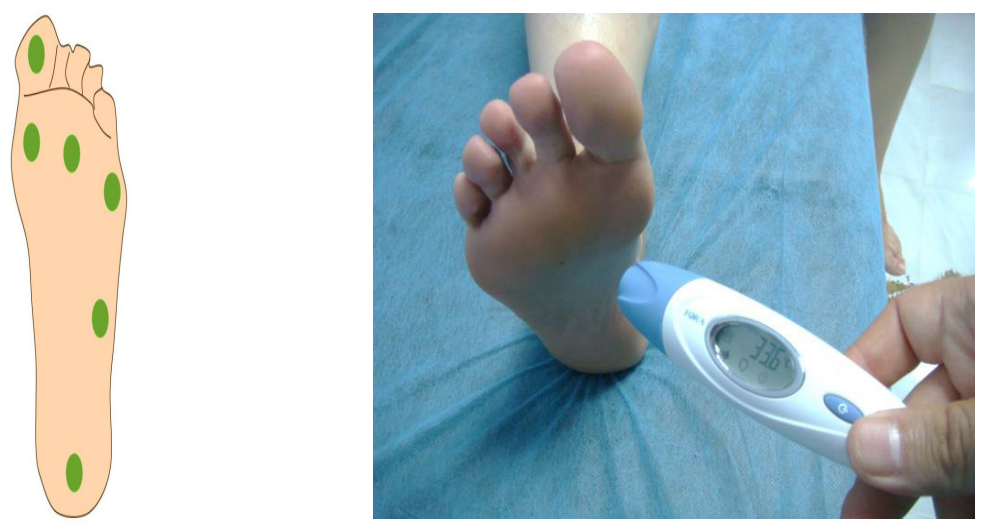

Figura 9. En la izquierda, puntos de aplicación de la temperatura a nivel plantar: $1^{\circ}$ dedo, cabezas de $1^{\circ}, 3^{\circ}$ y $5^{\circ}$ metatarsiano, arco longitudinal externo y centro del talón. En la derecha, aplicación perpendicular a la piel del termómetro por infrarrojos.

Es importante destacar que los termómetros dérmicos manuales, basados en tecnología de infrarrojos, han sido validados para la prevención de úlceras recurrentes ${ }^{25}$.

$\mathrm{Se}$ ha de tener en cuenta que cualquier zona con una temperatura mayor a $0^{\circ} \mathrm{C}$ emite radiación infrarroja. Sin embargo, sólo registran información de la temperatura de la piel, no de mayor profundidad ${ }^{26}$. En su estudio, Lazo - Porras et al (2016), los pacientes diabéticos se autocontrolan la temperatura en sus casas, siendo reforzados con recordatorios mediante SMS y mensajes de voz, para que lleven a cabo dichas tareas de prevención ${ }^{27}$. (Figura 10).
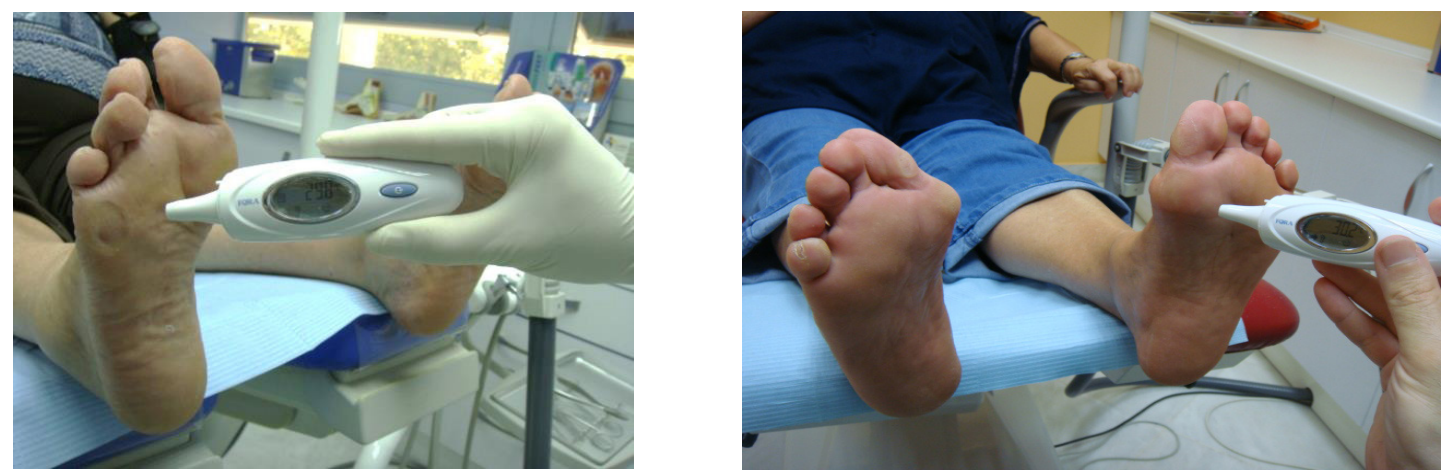

Figura 10. Aplicación del termómetro por infrarrojos a nivel metatarsal plantar de la cabeza del $5^{\circ}$ metatarsiano derecho y del $1^{\circ}$ metatarsiano izquierdo. 


\subsection{Termometría. Báscula térmica.}

Otro sistema empleado para la termometría del pie es la báscula térmica. Es una báscula con cuatro sensores térmicos (dos sensores delanteros y dos sensores traseros, en total

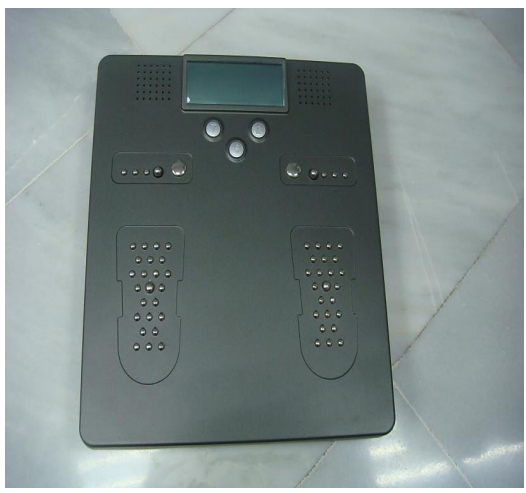

dos sensores para cada pie) que indica el peso, el índice de grasa corporal y la temperatura media de cada pie.

Actualmente, su uso es extendido en sujetos con Diabetes Mellitus en Alemania, Bélgica y Holanda $^{2}$ (Figura 11).

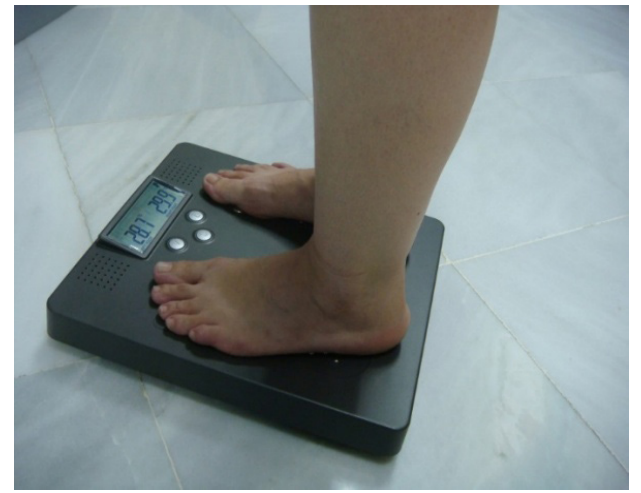

Figura 11. En la izquierda, la báscula térmica. En la derecha, procedimiento de medición de la temperatura plantar con báscula térmica.

Es un instrumento fácil de aplicar, permite obtener la temperatura de cada pie el peso e índice de masa corporal del sujeto (Figura 12). Se puede presentar alguna dificultad en casos de sujetos con pies de tamaños grandes, en los que

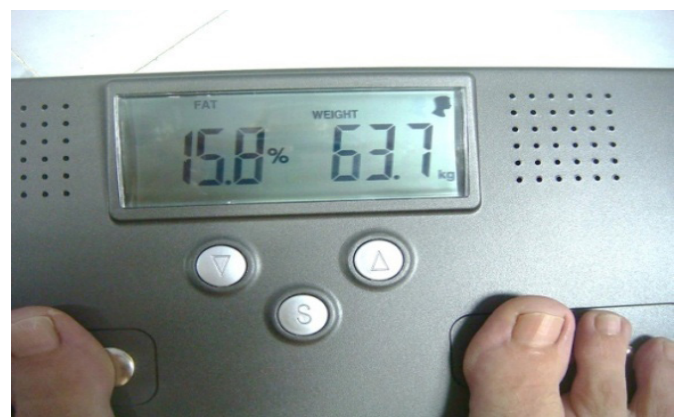

los sensores no quedan bien ubicados en la zona de medición planta ${ }^{3}$. Por otro lado, nos unimos a la observación de Roback (2010) respecto a que sería recomendable aumentar el número de sensores para cubrir todas las áreas plantares ${ }^{16}$.

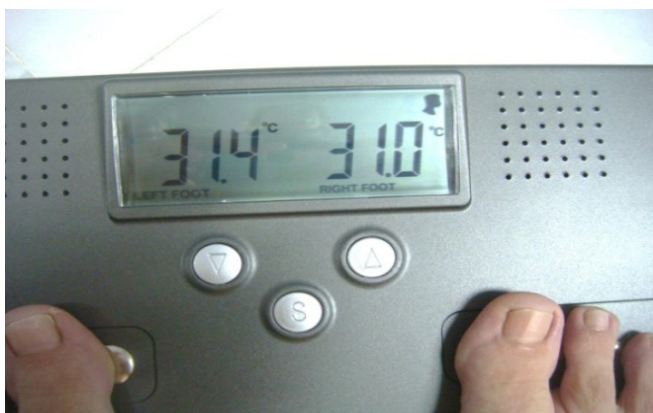

Figura 12. En la izquierda, valores de peso e índice de masa corporal, cuantificados con la báscula térmica. En la derecha, valores de la temperatura en cada pie, medidos con báscula térmica.

\subsection{Termometría. Termómetro con efecto Peltier.}

El termómetro de efecto Peltier consta de un lápiz con sonda que mide la temperatura cutánea en el dorso del pie del sujeto ${ }^{28,29}$.

Permite calcular el umbral de percepción térmica, tanto a la temperatura de frío como de calor, a partir de la temperatura basal de la piel.
Dispone de un martillo térmico con dos caras termocalentadas:

- La cara amarilla del martillo registra la temperatura basal.

- La cara verde del martillo registra la temperatura modificada a partir de la temperatura basal (de frío o calor) para valorar la percepción de dicho cambio de temperatura. 
- Los puntos de aplicación son en el dorso y en las zonas plantares de las cabezas de I y $\mathrm{V}$ metatarsianos.

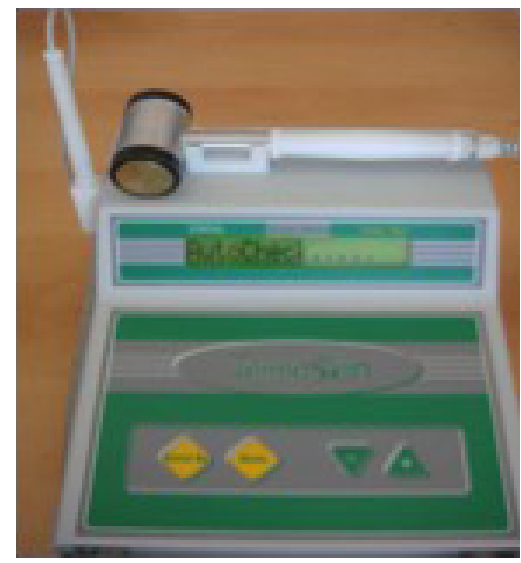

Se considera que existe presencia de neuropatía periférica diabética si no se percibe un incremento de $2^{\circ} \mathrm{C}$ respecto a la temperatura basal en la cabeza del I o V metatarsianos, a nivel plantar, en uno de los pies (García - De La - Peña et al, 2014) (Figuras 13 y 14).

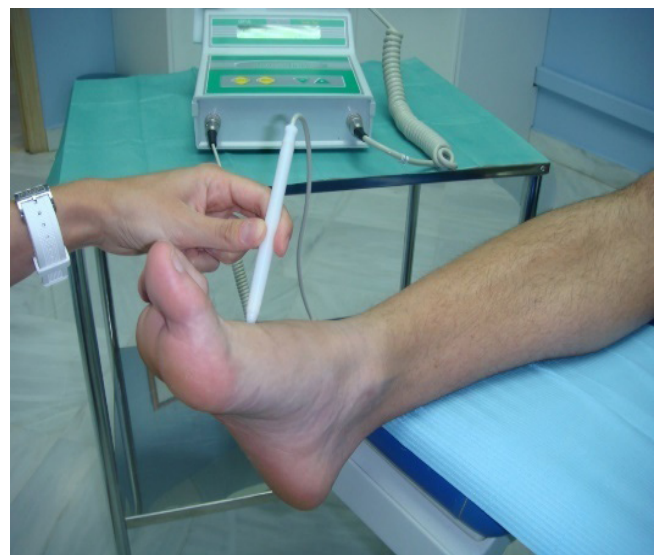

Figura 13. En la izquierda, termómetro basado en el efecto Peltier. En la derecha, medición de la temperatura podal en el dorso del pie con la sonda térmica del termómetro con efecto Peltier.
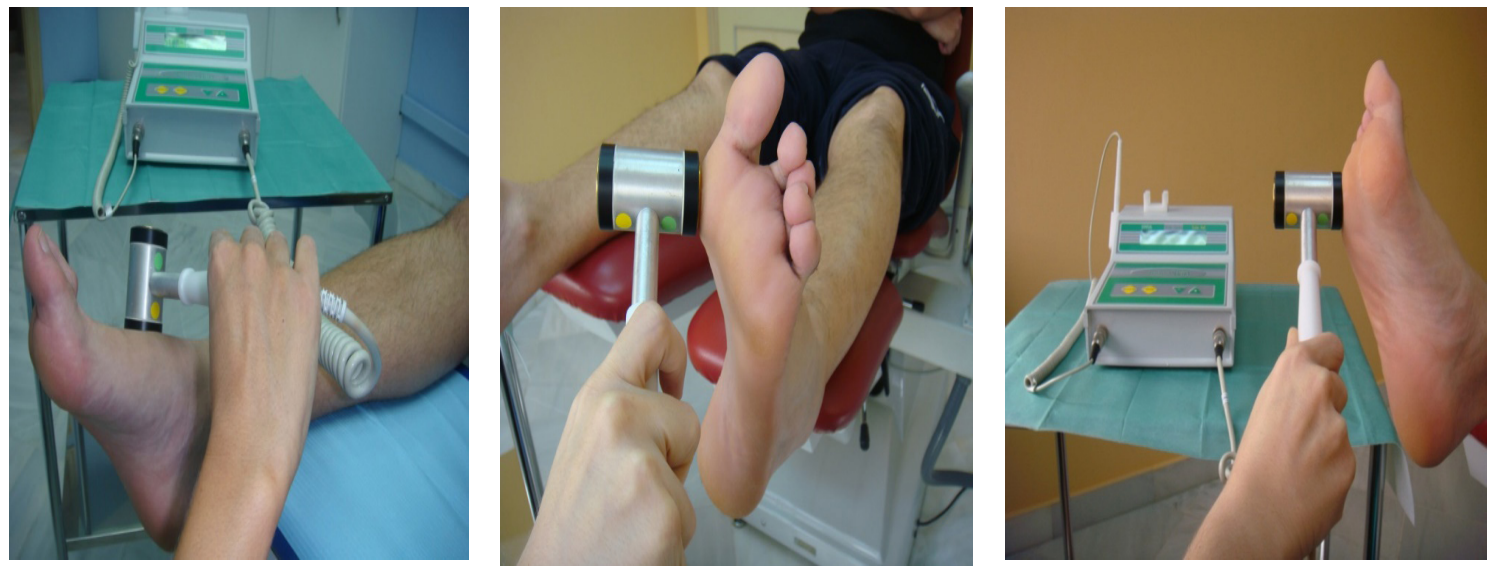

Figura 14. Medición de la percepción térmica con el martillo térmico en el dorso del pie (imagen de la izquierda) y en la zona plantar de las cabezas de $1^{\circ}$ (imagen del centro) y $5^{\circ}$ (imagen de la derecha) metatarsianos.

Para realizar las mediciones de la temperatura, es importante la temperatura ambiente en el momento de la medición ${ }^{23}$. La sala de valoración térmica debe estar a una temperatura de $24^{\circ} \mathrm{C} \pm 1,5^{\circ} \mathrm{C}$ y una humedad relativa $<50 \%{ }^{30}$.
Se debe realizar la aclimatación previa del sujeto, permaneciendo para ello descalzo y en reposo durante 15 minutos ${ }^{31}$.

Se ha de tener presente que si un sujeto camina antes de efectuarse la medición, este hecho tendrá influencia en los resultados, ya que 6 minutos caminando suponen un incremento de la temperatura plantar de $5^{\circ} \mathrm{C}^{32}$. 
5. ¿Por qué valorar la temperatura de los pies en la Diabetes Mellitus?

Las úlceras diabéticas generan un alto coste económico, una elevada carga personal y conllevan una importante trascendencia a nivel social, tanto el proceso en el que la lesión está presente como tras la amputación sufrida después de una nefasta evolución de ésta (Figura 14). Sin embargo, su prevención es sencilla, económica y basada en una evidencia científica que la sustenta.
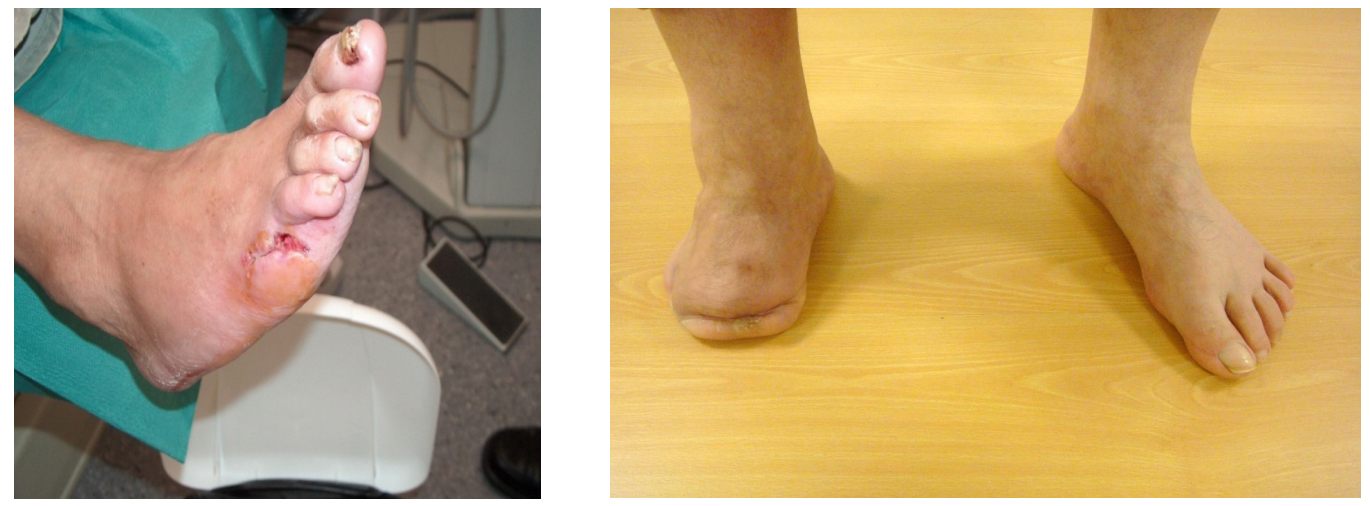

Figura 14. En la izquierda, ulceración en el lecho de la herida, consecuencia de la amputación del $5^{\circ}$ dedo del pie derecho. En la derecha, amputación a nivel transmetatarsal del pie derecho.

La prevención posee gran repercusión ya que el $75 \%$ de las úlceras plantares se pueden prevenir. A pesar de ello, por cada euro gastado en prevención de úlceras plantares por Diabetes Mellitus, 10 euros se emplean en su curación ${ }^{3}$. Un dato a tener en cuenta es que después de la aparición de la primera úlcera plantar en un paciente con Diabetes Mellitus, el riesgo de recurrencia se sitúa entre el $30-87 \%$ debido a la NPD ${ }^{15}$.

Los argumentos que justifican la inclusión de la valoración térmica en los protocolos de exploración neurológica de los pies de los sujetos con Diabetes Mellitus, son los siguientes:

1. En primer lugar, hemos de considerar que la prevención del Pie Diabético se basa, actualmente, en la exploración mediante dos pruebas sensitivas cualitativas con el monofilamento de Semmens - Weistein 5.07 y el diapasón graduado de Rydel - Scheiffer $128 \mathrm{~Hz}$, la autoinspección por parte del paciente y la palpación de pulsos, no estando la exploración de la temperatura incluida ${ }^{2}$.

Van Netten et al (2016) realizaron una revisión sistemática para analizar la efectividad de las intervenciones preventivas de aparición de úlceras en la Diabetes Mellitus, resultando más eficaz la medición de la temperatura de la piel de los pies (1 vez al día), consejos y calzado preventivos. Dichos autores refuerzan la sugerencia de la inclusión de la medición de la temperatura por el escaso tiempo de aplicación en el cribado exploratorio respecto al calzado preventivo ${ }^{33}$.

Es relevante la importancia de la termorresistencia de los materiales de la suela del calzado en los sujetos con Diabetes Mellitus ya que la percepción térmica se encuentra alterada en ellos, y se debe tener en cuenta que una temperatura mayor a $44^{\circ} \mathrm{C}$ genera una quemadura en la piel, con una temperatura de $55^{\circ} \mathrm{C}$, la quemadura se produce en 20 segundos y mientras, que si la temperatura es mayor a $60^{\circ} \mathrm{C}$, sólo son necesarios 3 segundos para que se desarrolle la quemadur ${ }^{34}$. (Figura 17).

2. El autocontrol de la temperatura (valoración del mismo punto en 2 pies) disminuye la tasa de aparición de úlceras ${ }^{35}$ (tabla 2).

En el estudio de Lavery y colaboradores (2004), el porcentaje de ulceraciones fue del $20 \%$ en sujetos sin autocontrol y del $2 \%$ en sujetos con control de la temperatura ${ }^{36}$. También es menor en el estudio del mismo autor en el año 2007, 29,3\% frente al 8,5\%. Armstrong y colaboradores (2007), también obtuvieron una menor incidencia de 
ulceraciones cuando se aplicó autocontrol de la temperatura ${ }^{37}$.
Además, en caso de que al control de la temperatura se añada una actuación rápida, la incidencia puede bajar al $60 \%{ }^{24}$.

\begin{tabular}{|c|c|c|}
\hline AUTORES & Autocontrol $\mathrm{T}^{\mathrm{a}}$ & Incidencia de ulceraciones \\
\hline \multirow{2}{*}{ Lavery et al, 2004 (36) } & NO & $20 \%$ \\
\hline & SI & $2 \%$ \\
\hline \multirow{2}{*}{ Lavery et al, 2007 (38) } & NO & $29,3 \%$ \\
\hline & SI & $8,5 \%$ \\
\hline \multirow{2}{*}{$\begin{array}{l}\text { Armstrong et al, } 2007 \\
\text { (37) }\end{array}$} & NO & $12,2 \%$ \\
\hline & SI & $4.7 \%$ \\
\hline
\end{tabular}

Tabla 2. Resumen de autores, con la incidencia de tasa de ulceraciones si se realiza o no el autocontrol de la temperatura. $\mathrm{T}^{\mathrm{a}}$ : temperatura.

3. Existe un intervalo de temperatura, diferencia de $2,2^{\circ} \mathrm{C}$ entre los dos pies, que sirve para controlar la aparición de lesiones propias del Pie Diabético ${ }^{39}$.

Este intervalo, como prueba diagnóstica de infección, presenta una especificidad del $25 \%$ y una sensibilidad del $80 \%{ }^{40}$.

Con un intervalo de diferencia de temperatura de $1,35^{\circ} \mathrm{C}$ se considera que es necesaria una actuación urgente temperatura ${ }^{39}$.

4. Otro argumento es que la valoración de la temperatura de los pies es una técnica eficaz y no invasiva. Su aplicabilidad es viable, tal y como se pilotó en Noruega en el estudio de Shafjed y colaboradores $(2015)^{15}$. En Australia es el principal método de cribado en el pie de riesgo ${ }^{12}$.

La termografía es útil en la detección de úlceras plantares con osteomielitis ${ }^{41}$. A nivel práctico, el control de la temperatura es un parámetro efectivo para controlar la consolidación ósea y el proceso evolutivo de la enfermedad de Charcot, permitiendo una retirada segura de la inmovilización ${ }^{42}$ (Figura 15).
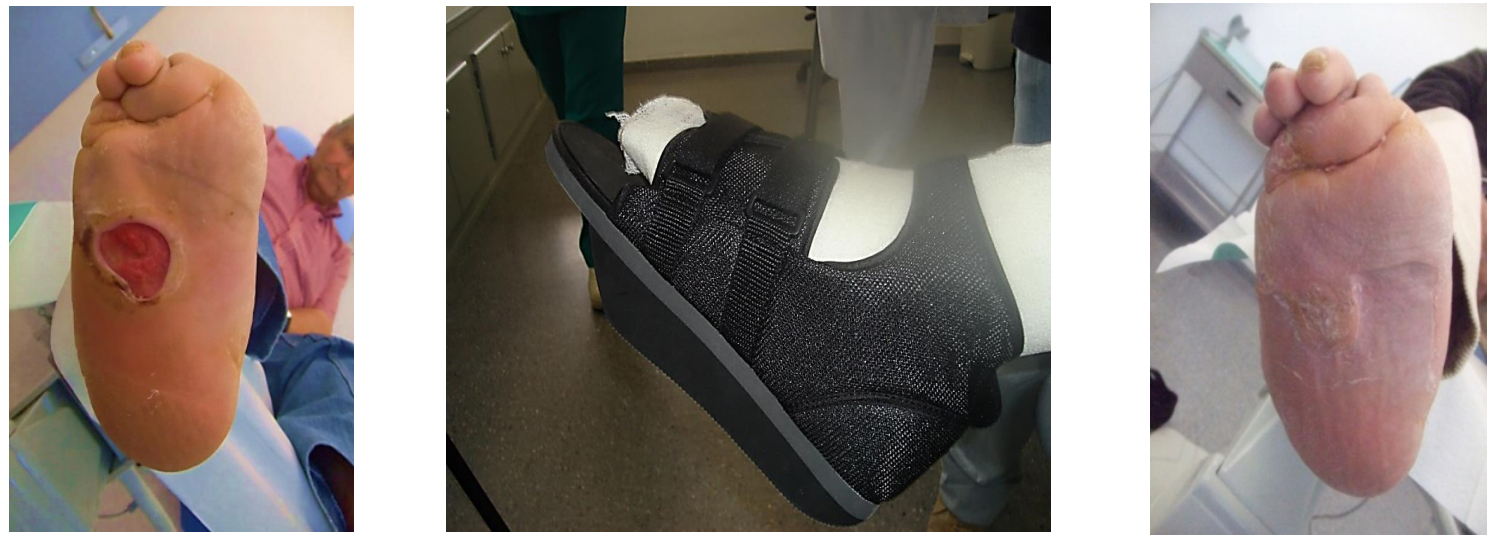

Figura 15. En la izquierda, úlcera plantar en mediopié derecho en un sujeto con artropatía de Charcot. En la imagen del centro, aplicación de zapato de descarga como parte del tratamiento. En la derecha, resolución de la lesión. 
A pesar de la evidencia científica al respecto, la evaluación de la temperatura en pacientes diabéticos no se aplica, posiblemente por la falta de resultados clínicos o la escasa difusión de esos resultados. Puede que no se haya experimentado en zonas geográficas próximas, con temperaturas ambientales similares, o bien que la adhesión del paciente al autocontrol de la temperatura no sea la óptima.

Respecto al futuro, la tendencia para evaluar este parámetro pueden ser instrumentos con almacenamiento automático de los datos procesados, con obtención inmediata del cálculo de las diferencias de temperatura, con envío de los datos registrados al facultativo y existirá calcetines con sensores de temperatura, similares a los que se pueden encontrar actualmente para uso en bebes o en otros prototipos.

\section{Conclusiones}

Las conclusiones del presente trabajo son las siguientes:

1. Una diferencia de $2,2^{\circ} \mathrm{C}$ en el mismo punto de ambos pies de un sujeto, indica la aparición de lesiones propias del Pie Diabético, bien una ulceración si hay lesión, o una artropatía de Charcot.

2. La medición de la temperatura de los pies puede realizarse mediante la termografía y la termometría.

3. La evidencia científica indica que el control de la temperatura de los pies disminuye la tasa de ulceraciones en la Diabetes Mellitus.

4. Abogamos por la inclusión de la valoración térmica en el protocolo de exploración de los sujetos con Diabetes Mellitus, debido a su efectividad en la prevención de ulceraciones así como por su escaso tiempo de cribado.

\section{Bibliografía}

1. Chicharro E, Portabales I, Ramírez L, Torrent E. Monitorización de la temperatura del pie como herramienta en la neuropatía diabética. Rev Int Ciencias Podol. 2016; 10 (1): 9 - 16.

2. Medrano R, Pera G, Gil E, Valverde I, García O, Medrano B. Termometría plantar y pie de riesgo en Atención primaria. Estudio termopiedi. Nure (Inv). 2015; 12 (77): 1 - 13.

3. Bus S, van Netten J. A shift in priority in diabetic foot care and research: $75 \%$ of foot ulcers are preventable. Diabetes Metab Res Rev. 2016; 32 (Suppl. I): 195 - 200.

4. Herrera MP, Portela KR, Tortotici V, Navas T, Garcés C, Santiago J. Neuropatía diabética periférica: eficacia clínica de la evaluación semiológica. Med Interna (Caracas). 2015; 31 (2): 82 - 101.

5. ADA. 68th Scientific Sessions. California (USA), 6 - 10 June 2008.

6. Tanenberg RJ, Donofrío PD. Problemas neuropáticos de los miembros inferiores en los pacientes diabéticos. En: Bowker JH, Pfeifer MA. Levin y O'Neil. El pie diabético. $7^{\mathrm{a}}$ Ed. Barcelona: Elsevier Mosby; 2008. p. $33-74$.

7. Vinik AI, Ullal J, Parson HK, Casellini CM. Diabetic neuropathies clinical manifestations and current treatment options. Nat Clin Pract Endocrinol Metab. 2006; 2 (5): 269 - 81.

8. Goller H, Lewis DW, McLaughlin RE. Thermographic studies of human skin subjected to localized pressure. Am J Roentgenol. 1971; 113 (4): 749 - 54.

9. Bagavathiappan S, Jayakumar T, Rao PN, Varalakshmi M, Mohan V. Correlation between plantar foot temperatura and diabetic neuropathy: a case study by using an infrared termal imaging technique. $\mathrm{J}$ Diabetes Sci Technol. 2010; 4 (6): 1386 - 92.

10. Papanas N, papatheodorou K, Papazoglou D, Monastiriotis C, Maltezos E. Foot temperatura in type 2 diabetic patients with or without peripheral neuropathy. Exp Clin Endocrinol Diabetes. 2009; 117 (I): $44-7$.

11. García - De - La - Peña R, Benhamú S, Munuera PV. Sensitivity and Specitivity of a new test for Thermographic Evaluation of the Foot in the Diagnosis of Diabetic Peripheral Polyneuropathy. Adv Skin Wound Care. 2014 Nov; 27 (II): 491 - 8.

12. Houghton V, Bower V, Chant D. Is an increase in skin temperatura predictive of neuropathic foot ulceration in people with diabetes? A systematic review and meta - analysis. J Foot Ankle Res. 2013; 6 (I): 31 .

13. Armstrong DG, Lipsky BA, Polis AB, Abramson MA. Does dermal thermometry predict clinical outcome in diabetic foot infection. Analysis of data from the SIDESTEP trial. Int Wound J. 2006; 3 (4): 302 - 7. 
14. Bus S. Innovations in plantar pressure and foot temperature measurements in diabetes. Diabetes Metab Res Rev. 2016; 32 (Suppl. 1): $221-6$.

15. Skafjeld A, Iversen MM, Holme I, Ribu L, Hvaal K, Kilhovd BK. A pilot study testing the feasibility of skin temperature monitoring to reduce recurrent foot ulcers in patients with diabetes - a randomized controlled trial. BMC Endocr Disord. 2015; 15: 55 - 62.

16. Roback K. An overview of temperature monitoring devices for early detection of diabetic foot disorders. Expert Med Devices. 2010; 7 (5): $711-8$.

17. Sibbald RG, Mufti A, Armstrong DG. Infrared skin thermometry: an underutilized cost-effective tool for routine wound care practice and patient high-risk diabetic foot self-monitoring. Adv Skin Wound Care. 2015; 28 (1): $37-44$.

18. Najafi B, Wrobel JS, Grewal G, Grewal G, Menzies RA, Talal TK, Zirie M, Armstrong DG. Plantar Temperature Response to Walking in Diabetes with and without Acute Charcot: The Charcot Activity Response Test. J Aging Res. 2012; 2012:140968.

19. Carrascosa E. Descripción de las variables que participan en el desarrollo de la neuroartopatía de Charcot en una muestra de pacientes. Libro de Actas 38 Congreso Nacional de Podología. Bilbao, 2007.

20. Mori T, Nagase T, Takehara K, Oe M, Ohashi Y, Amemiya A et al. Morphological pattern classification system for plantar thermography of patients with diabetes. J Diabetes Sci Technol. 2013; 7 (5): $1102-12$.

21. van Netten JJ, van Baal JG, Liu C, van der Hejiden F, Bus SA. Infrared thermal imaging for automated detection of diabetic foot complications. J Diabetes Sci Technol. 2013; 7 (5): 1122 - 9.

22. Balbinot LF, Robinson CC, Achaval M, Zaro MA, Brioschi ML. Repeatability of infrared plantar thermography in diabetes patients: a pilot study J Diabetes Sci Technol. 2013; 7 (5): 1130 - 7.

23. Liu C, van Neten JJ, van Baal JG, Bus SA, van der Heijden F. Automatic detection of diabetic foot complications with infrared thermography by asymmetric analysis. J Biomed Opt. 2015; 20 (2): $26003-1-10$.

24. Pafili K, Papanas N. Thermography in the follow up of the diabetic foot: best to weigh the enemy more mighty than he seems. Expert Rev Med Devices. 2015; 12 (2): 131 - 3.

25. Bharara M, Schoess J, Nouvong A, Armstrong DG. Wound inflammatory index: a "proof of concept" study to assess wound healing trajectory. J Diabetes Sci Technol. 2010 Jul 1; 4 (4): 773 - 9.

26. Staffa R, Bernard V, Kubicek L, Vlachovsky R, Vlk D, Mornstein V, Staffa R. Using Noncontact Infrared Thermography for Long-term Monitoring of Foot Temperatures in a Patient with Diabetes Mellitus. Ostomy Wound Manage. 2016; 62 (3): 54 - 61.

27. Lazo - Porras et al. Implementation of foot thermometry plus mHealth to prevent diabetic foot ulcers: study protocol for a randomized controlled trial. Trials. 2016; 17 (1): $206-16$.

28. García R. Valoración termográfica podal en el diagnóstico de la neuropatía periférica diabética. [Tesis Doctoral]. Sevilla: Universidad de Sevilla; 2012.

29. Bottini P, Galetta M, Meloncelli I, Clementi L, Vespasiani G. Thermoskin: un nuovo strumento per la valutazione della sensibilitá termica nei pazienti con diabete. Giornale Italiano di Diabetologia e Metabolismo. 2003; 23: $11-6$.

30. García UO, Vera A, Cortela G, Negreira C, Leija L. Comparison of diabetic foot diagnosis between thermography infrared methods and the elastography techniques. Global Medical Engineering Physics Exchanges/Pan American Health Care Exchanges (GMEPE/PAHCE). 2016.

31. Vilcahuaman L, Harba R, Canals R, Zequera M, Wilches C, Arista MT et al. Detection of diabetic foot hyperthermia by infrared imaging. Conf Proc IEEE Eng Med Biol Soc. 2014; 2014: 4831 -4 .

32. Yavuz M, Brem RW, Davis BL, Patel J, Osbourne A, Matassini MR et al. Temperature as a predictive tool for plantar triaxial loading. J Biomech. 2014; 47 (15): 3767 - 70.

33. van Netten JJ, Price PR, Lavery LA, Monteiro - Soares M, Rasmussen A, Jubitz Y, Bus SA. Prevention of foot ulcers in the at - risk patient with diabetes: a systematic review. Diabetes Metab Res Rev. 2016; 32 (Suppl. 1): 84 - 98.

34. Clifton T, Khoo TW, Andrawos A, Thomson S, Greenwood JE. Variation of surface temperatures of different ground materials on hot days: Burn risk for the neuropathic foot. Burns. 2016; 42 (2): 453 - 6.

35. Shirazi A, Nasiri M, Yazdanpanah. Dermatological and musculoskeletal assessment of diabetic foot: A narrative review. Diabetes Metab Syndr. 2016; 10 (2 Suppl 1): S158 - 64.

36. Lavery LA, Higgins KR, Lanctot DR, Constantinides GP, Zamorano RG, Armstrong DG, et al. Home monitoring of foot skin temperatures to prevent ulceration. Diabetes care. 2004; 27 (11): 2642 - 7.

37. Armstrong DG, Holtz - Keiderer K, Wendel C, Mohler MJ, Kimbriel HR, Lavery LA. Skin temperature monitoring reduces the risk for diabetic foot ulceration in high - risk patient. Am J Med. 2007; 120 (12): $1042-6$. 
38. Lavery LA, Higgins KR, Lanctot DR, Constantinides GP, Zamorano RG, Athanasiou KA, et al. Preventing diabetic foot ulcer recurrence in high - risk patients. Diabetes care. 2007; 30(1): $14-20$.

39. van Netten JJ, Prijs M, van Baal JG, Liu C, van der Heijden F, Bus SA. Diagnostic values for skin temperature assesment to detect diabetes - related foot complications. Diabetes Technol Ther. $2014 ; 16$ (11): $714-21$.

40. Hazenberg CE, van Netten JJ, van Baal JG, Bus SA. Assesment of signs of foot infection in diabetes patients using photographic foot imaging and infrared themography. Diabetes Technol Ther. $2014 ; 16$ (6): $370-7$.

41. Oe M, Yotsu RR, Sanada H, Nagase T, Tamaki T. Thermographic findings in case of type 2 diabetes with foot ulcer and osteomyelitis. J Wound Care. 2012; 21 (6): 274, 276 -8.

42. Moura - Neto A, Fernandes TD, Zantut - Wittmann DE, Trevisan RO, Sakaki MH, Santos AL et al. Charcot foot: skin temperature as a good clinical parameter for predicting disease outcome. Diabetes Res Ckin Pract. 2012; 96 (2): e11 - 4. 